Cahiers
Recherche
surles Droits Fondamentaux
Cahiers de la recherche sur les droits fondamentaux

17 | 2019

La motivation des actes administratifs. Le droit français à la lumière du droit administratif comparé

\title{
La motivation de l'acte administratif en Argentine
}

Pedro Aberastury

\section{(2) OpenEdition}

Journals

Édition électronique

URL : https://journals.openedition.org/crdf/3810

DOI : $10.4000 /$ crdf.3810

ISSN : 2264-1246

Éditeur

Presses universitaires de Caen

Édition imprimée

Date de publication : 31 décembre 2019

Pagination : 153-160

ISBN : 978-2-84133-960-0

ISSN : $1634-8842$

Référence électronique

Pedro Aberastury, «La motivation de l'acte administratif en Argentine », Cahiers de la recherche sur les droits fondamentaux [En ligne], 17 | 2019, mis en ligne le 06 février 2021, consulté le 16 novembre 2022. URL : http://journals.openedition.org/crdf/3810 ; DOI : https://doi.org/10.4000/crdf.3810 


\title{
La motivation de l'acte administratif en Argentine
}

\author{
Pedro ABERASTURY \\ Professeur titulaire adjoint de droit administratif à la faculté de droit de l'université de Buenos Aires (Argentine)
}

\author{
I. L'élément de motivation \\ II. La motivation en tant que principe général \\ III. Les actes qui doivent être motivés \\ IV. Conditions requises pour la motivation: le contenu de la motivation \\ V. Les exceptions au principe de motivation des actes. Les actes automatisés
}

Dès la promulgation de la Constitution nationale de $1853^{1}$, la République argentine a adopté la forme de l'État fédéral, les provinces conservant leur autonomie pour toutes les matières non déléguées au gouvernement fédéral. Elle a ainsi suivi le modèle de la Constitution des États-Unis de 1787 , avec quelques adaptations particulières telles que l'interdiction expresse faite au pouvoir exécutif de la nation d'exercer la fonction juridictionnelle (art. 116 de la Constitution nationale), en réservant cette faculté à la Cour suprême de justice de la nation (CSJN), tribunal impartial et indépendant, en tant que tribunal supérieur. L'intervention de cette Cour est conditionnée par l'existence d'un procès et l'État doit se conformer aux décisions de la haute juridiction qui a compétence pour statuer sur toutes les affaires. La Constitution fédérale prévoit que les provinces conservent l'ensemble des pouvoirs non délégués à l'État fédéral et que chaque province sanctionne sa propre Constitution, en tenant compte du système représentatif, républicain et fédéral et les principes, déclarations et garanties de la Constitution nationale.

Le contrôle de constitutionnalité est diffus, étant donné que le système juridique argentin ne prévoit pas l'existence d'une Cour constitutionnelle. C'est ainsi que tout juge provincial ou fédéral peut statuer sur l'inconstitutionnalité de la loi et des actes administratifs émanant des organes de l'État fédéral ou des provinces, en réservant à la CSJN le contrôle ultime desdites décisions par la voie du recours extraordinaire ${ }^{2}$.

Le contrôle de l'activité du pouvoir exécutif provincial demeure ainsi entre les mains de ses propres cours provinciales, malgré le contrôle final que la CSJN est susceptible d'effectuer.

La loi 19.549 (1972), dite loi nationale sur la procédure administrative (LNPA) ${ }^{3}$, a été adoptée il y a plus de quarante-cinq ans. Elle a représenté une étape remarquable en ce qui concerne la régulation de la procédure administrative dans le domaine de l'administration publique nationale. À leur tour, les provinces ont également adopté leurs propres normes de procédure administrative et, pour la plupart d'entre elles, les textes qui règlent l'acte administratif unilatéral restent similaires à la norme nationale.

La LNPA prévoit que, dans le cas d'existence d'une procédure administrative, la décision restera soumise au contrôle devant l'administration par le supérieur hiérarchique ainsi que dans le domaine de la juridiction judiciaire.

1. Constitution encore actuellement en vigueur, en ligne: http://servicios.infoleg.gob.ar/infolegInternet/anexos/o-4999/804/norma.htm.

2. Loi 48, art. 14, en ligne: http://servicios.infoleg.gob.ar/infolegInternet/anexos/1150oo-119999/116296/texact.htm.

3. Réglementée par le décret 1759/72, Règlement national sur la procédure administrative (RNPA). Loi disponible en ligne: http://servicios.infoleg. gob.ar/infolegInternet/anexos/200oo-24999/22363/norma.htm. 
En ce qui concerne le contrôle interne effectué dans le cadre de l'administration, en vertu du principe hiérarchique, les actes des subordonnés sont soumis au contrôle du supérieur hiérarchique et, conformément à ce principe, ce contrôle porte sur la légalité de l'action ${ }^{4}$, malgré la possibilité d'un contrôle d'opportunité, de mérite ou de convenance. C'est dans ce schéma que l'administration, dans le cadre de la procédure administrative, réalise le contrôle interne de son activité.

D'autre part, et une fois la voie administrative épuisée, il existe la possibilité de contester en justice les actes administratifs individuels et les actes réglementaires dans un délai de forclusion de quatre-vingt-dix jours ouvrables. C'est ainsi que l'action administrative reste contrôlée non seulement par voie de recours administratif mais aussi par voie de recours juridictionnel devant un organe impartial et indépendant. Dans ce dernier cas, le contrôle est toutefois limité au seul contrôle de légalité.

\section{L'élément de motivation}

D'après la terminologie de la LNPA, pour que l'acte administratif individuel soit valable, il est nécessaire que soient présents les éléments prévus dans la norme juridique. L'omission de l'un d'entre eux entraîne son illégalité. Au-delà des classifications, les éléments objectifs du droit argentin sont la compétence, la cause, la motivation, l'objet, la procédure, la forme et la finalité (le but), tandis que parmi les éléments subjectifs on trouve ceux qui relèvent de la volonté.

Il ne faut pas oublier que le principe de compétence s'applique à l'administration. Ce principe comprend le pouvoir d'agir', alors que pour le particulier c'est la garantie prévue par l'article 19 de la Constitution qui reste valable. Cette norme constitutionnelle prévoit que «Personne ne sera contraint de faire ce que la loi n'ordonne pas, ni privé de ce que la loi n'interdit pas ${ }^{6}$. Cela revient à dire que, tandis que l'administration nécessite une norme préalable d'autorisation ou implicitement incorporée dans le système juridique pour pouvoir agir dans le cadre de la légalité, le particulier jouit du principe de libre arbitre ou de liberté, sans avoir besoin d'une autorisation légale pour le développement de son activité, à condition de ne pas violer ou transgresser les normes juridiques.

La doctrine a classifié les éléments de l'acte administratif, ce qui explique que le recensement que nous venons d'effectuer ne signifie pas que les uns fassent partie ou soient la conséquence des autres. Cet article n'abordera que la motivation de l'acte administratif. Avant de continuer, nous tenons à signaler qu'il s'agit d'une exigence formelle et nécessaire dont l'inobservance peut entraîner la déclaration d'illégalité de l'acte administratif, devant les juridictions administratives comme judiciaires, c'est-àdire que le manque de motivation peut être soulevé à la fois dans le recours administratif et le recours juridictionnel.

Ceci explique que nous ferons référence à des décisions judiciaires confirmant ou déclarant illégal un acte administratif insuffisamment motivé ou avec une motivation non adéquate ou non raisonnable.

Comme nous l'avons signalé, au moment de l'adoption de la LNPA les conditions requises essentielles pour l'acte administratif ont été établies par l'article 7. Cependant, le législateur a employé les mots «conditions requises essentielles» et non pas «éléments essentiels » pour ceux qui sont objectifs. La doctrine s'est ensuite interrogée sur le point de savoir si ces " conditions requises essentielles» constituaient les éléments de l'acte, car l'article 8 fait référence à la forme et l'article 14 aux vices de la volonté (et, dans ce dernier cas, on énumère les conditions requises signalées à l'article 7 ).

Il existe par ailleurs deux types de nullité de l'acte administratif prévues par la LNPA. D'une part, l'article 14 prévoit la nullité absolue, pour sanctionner non seulement les vices de la volonté, mais également la violation des conditions prévues par les articles 7 et 8 . L'élément en cause est tellement affecté qu'il se trouve exclu de l'acte et l'acte doit être annulé. D'autre part, l'article 15 de la LNPA prévoit la nullité relative. L'acte est alors simplement susceptible d'être annulé, car l'élément en cause n'est pas affecté au point d'être exclu et l'acte peut être confirmé7. Le législateur a donc utilisé une terminologie différente pour renvoyer aux éléments de l'acte administratif. Il en découle que la motivation peut être considérée soit comme un élément autonome, soit comme le sous-élément d'un autre élément.

Il faut signaler que nous trouvons la notion de motivation dans le paragraphe e) de l'article 7 de la LNPA dans les termes suivants: l'acte «devra être motivé, et énoncer de manière concrète les motifs qui ont conduit à l'émission de l'acte, en signalant aussi les éléments exigés prévus au paragraphe b) du présent article». Le paragraphe b) fait référence à la cause de l'acte et il prévoit qu' «il devra s'appuyer sur des faits qui en seraient la cause et sur le droit applicable».

Il convient d'ajouter que le paragraphe c) prévoit deux situations: 1) les motifs qui mènent à l'émission de l'acte et; 2 ) l'extériorisation de la cause.

C'est ainsi que la doctrine a distingué entre motif et motivation. En effet, le motif signalé dans les «visas» de l'acte renvoie aux étapes de la procédure, et indique que toutes les démarches nécessaires à l'émission de l'acte ont été accomplies, c'est-à-dire que le fonctionnaire possédant la compétence requise pour l'acte est en mesure d'adopter l'acte administratif. Pour ce faire, il doit respecter la procédure essentielle et substantielle prévue au paragraphe d)

4. Art. 73 et 74 RNPA.

5. Art. 3 LNPA.

6. Toutes les traductions de l'espagnol au français ont été faites par l'auteur de cet article.

7. L'article 14 de la LNPA donne l'exemple d'un acte émis sans compétence en raison du degré mais où la délégation est possible. 
de l'article 7 de la LNPA. Ces exigences procédurales comprennent notamment l'obtention de l'avis conforme de l'organisme consultatif avant l'émission de l'acte. En outre, le respect de la procédure doit protéger le droit de la défense dans le cadre d'une procédure administrative, c'est-à-dire offrir au particulier visé par un acte défavorable la possibilité de discuter les griefs et de fournir les preuves nécessaires pour résoudre le cas.

Si le motif a pour objet de déterminer que la procédure s'est bien déroulée et que le dossier est prêt pour l'émission de l'acte administratif définitif - c'est-à-dire celui qui tranche les questions posées -, c'est parce que nous distinguons les actes administratifs définitifs de ceux qui ne le sont pas (simples actes de procédure), réservant, en principe, l'exigence de la motivation aux premiers.

Voilà pourquoi nous affirmons que, même si de prime abord l'on est tenté de les confondre, motif et motivation sont des notions distinctes. En effet, ils constituent l'un et l'autre des extériorisations aux finalités différenciées.

Le motif n'est qu'une vérification formelle de la conclusion de la procédure et de l'accomplissement des démarches nécessaires pour l'émission de l'acte. En revanche, la motivation revêt une plus grande importance étant donné, qu'à travers elle, il ne s'agit pas de constater l'adéquation des faits à la norme mais d'extérioriser l'existence d'un jugement de valeur de la part de l'administration ${ }^{8}$.

La motivation exige l'expression, dans l'acte administratif, des raisons prises en considération pour son émission, c'est-à-dire que la norme demande la mention concrète des faits qui constituent le fondement de la décision. C'est ainsi que la décision, contenue dans l'objet, sera une conséquence directe de l'analyse de ces faits et de ces antécédents, de sorte qu'on puisse suivre le raisonnement pour aboutir à la décision.

La LNPA a prévu un principe général selon lequel les actes administratifs définitifs, c'est-à-dire ceux qui tranchent les questions posées, doivent être motivés et l'absence de motivation rend de tels actes illégaux.

Cependant, même s'il existe des actes, en principe légitimes, pour lesquels l'absence de motivation est acceptée et pour lesquels différentes normes locales de procédure administrative n'exigent pas de manière expresse leur motivation, la LNPA ne fait pas de distinction entre les actes pour lesquels la motivation est exigée et ceux pour lesquels elle ne l'est pas.

\section{La motivation en tant que principe général}

En Argentine, avant l'adoption de la LNPA, la doctrine nationale avait signalé l'existence d'un principe général d'obligation de motivation les actes. Le principe trouvait son fondement dans la forme républicaine du gouvernement $^{9}$, dans le régime démocratique ${ }^{10}$ ou dans le souci de dissiper «toute ombre d'arbitraire ${ }^{11}$. Après l'adoption de la LNPA, la doctrine a repris cette orientation ${ }^{12}$, afin de garantir le respect des principes de légalité et de l'action raisonnable et le respect du droit à une procédure régulière ${ }^{13}$, et elle a considéré ce principe comme un facteur de protection des particuliers face à l'action de l'État.

En droit comparé, nous trouvons l'exigence de motivation en tant que principe général dans la loi allemande de procédure administrative - Verwaltungsverfahrensgesetz (VwVfG) - du 25 mai 1976. L'acte administratif écrit ou électronique, ainsi que l'acte administratif confirmé par écrit ou par voie électronique, doivent être justifiés, en application du principe général prévu par le $\$ 39$. En outre, le $\$ 39$ I 2 signale que la justification doit comprendre les considérations essentielles de fait et de droit qui ont amené l'autorité administrative à prendre une telle décision, et les critères déterminant l'exercice du pouvoir discrétionnaire ( $\$ 39$ I 3 ). Le $\$ 39$ présente donc un principe général de justification de l'acte individuel.

La situation du droit français est différente étant donné les résistances face à l'exigence de motivation. Après l'évolution jurisprudentielle due à l'arrêt Barel $^{14}$, le législateur a opté pour le principe contraire dans la loi de 1979 codifiée à l'article L. 211-2 du Code des relations entre le public et l'administration (CRPA) ${ }^{15}$. Par conséquent, nous pouvons dire, a contrario sensu, que l'énumération prévue dans la loi générale de 1979 ou dans les lois spéciales est exhaustive, c'est-à-dire que pour les cas non prévus l'exigence de motivation n'existe pas.

De plus, en droit comparé latino-américain, il existe un principe général qui exige la motivation des actes administratifs. C'est Pedro José Jorge Coviello ${ }^{16}$ qui arrive à cette conclusion d'après l'analyse de différents systèmes juridiques latino-américains, tels que, entre autres, ceux en vigueur dans la république orientale de l'Uruguay, dans la république du Costa Rica, et dans la république fédérative du Brésil. En droit comparé ibéro-américain, l'exigence de motivation est prévue dans la plupart des

8. J. C. Cassagne, Derecho administrativo, Buenos Aires, LexisNexis, 2006, t. II, p. 176.

9. R. Bielsa, Derecho administrativo, $6^{\mathrm{e}}$ éd., Buenos Aires, La Ley, 1964, t. II, p. 85 sq.

10. B. A. Fiorini, Manual de derecho administrativo, Buenos Aires, La Ley, 1968, t. I, p. 340 sq.

11. M. S. Marienhoff, Tratado de derecho administrativo, $4^{\mathrm{e} e ́ d ., ~ B u e n o s ~ A i r e s, ~ A b e l e d o ~ P e r r o t, ~ 2011, ~ t . ~ I I, ~ p . ~} 263$.

12. A. Gordillo, Tratado de derecho administrativo, $9^{\mathrm{e}}$ éd., Buenos Aires, Fundación de derecho administrativo, 2007, t. III, p. X-16.

13. G. S. Tawill, L. M. Monti, La motivación del acto administrativo, Buenos Aires, Depalma, 1998, p. 10 sq.

14. CE, 28 mai 1954, Barel, Recueil Lebon, p. 308, concl. Letourneur; J.-M. Auby, R. Drago, Traité de contentieux administratif, Paris, LGDJ, 1962, t. II, p. 590. Si le gouvernement refuse de communiquer au Conseil d'État la motivation de sa décision, l'acte est considéré comme irrégulier.

15. Ordonnance $\mathrm{n}^{\circ} 2015-1341$ du 23 octobre 2015.

16. P. J. J. Coviello, «La motivación del acto administrativo como medio de control judicial del ejercicio de las potestades discrecionales», in El control de la actividad estatal, faculté de droit, université de Buenos Aires, 2016, en ligne: http://www.derecho.uba.ar/docentes/libro-el-control-de-laactividad-estatal.php. 
cas comme un principe général, afin de sauvegarder la légalité administrative.

Ce principe général comporte toutefois des exceptions. Certes, il est exact que l'exigence de motivation pour les actes administratifs est en relation avec la légalité de l'action de l'administration et se rapporte à la sauvegarde des droits des particuliers. Néanmoins, même Miguel S. Marienhoff, dont les idées ont été suivies par les rédacteurs de la LNPA, a reconnu que, pour certains actes, la motivation n'était pas essentielle. Tel est le cas des actes verbaux. Il reste que la détermination des actes dont la nature exige la motivation est imprécise ${ }^{17}$.

Par ailleurs, la question a été posée de savoir si la motivation faisait partie de l'exigence de «forme», car, si tel était le cas, nous pensons qu'elle ne pourrait pas se trouver in aliunde ${ }^{18}$, c'est-à-dire en dehors du dossier où l'acte est prononcé, mais s'il est fait renvoi à un autre dossier, le renvoi doit être réalisé de manière expresse pour être validé. L'absence de renvoi vicie l'acte ${ }^{19}$. Si la motivation fait défaut ou si elle est insuffisante, l'acte ne peut pas être reformulé par une motivation a posteriori ${ }^{20}$, dans la mesure où il s'agit d'une exigence essentielle. Cependant, il existe des exceptions, comme nous le verrons plus tard.

\section{Les actes qui doivent être motivés}

Nous avons rattaché la motivation à la légalité «formelle», et, avant d'aborder les conditions de sa formulation, nous devons l'analyser en relation avec l'exercice des pouvoirs de l'administration. Il convient de distinguer l'exercice de la compétence liée de celui du pouvoir discrétionnaire par l'administration. Dans le premier cas, l'acte sera motivé s'il s'appuie sur l'existence des faits prévus par le législateur et considérés importants et nécessaires pour la prise de décision. C'est-à-dire que, lorsque l'administration doit adopter une décision qui est la seule possible, elle sera contrainte au moment de la prise de décision puisque le législateur a déjà prévu l'attitude à suivre lorsque les conditions requises sont remplies. La motivation ne se manifestera donc que dans l'expression de l'observation des conditions posées. Dans le cas d'un pouvoir discrétionnaire, il faudra exprimer le raisonnement suivi et la proportionnalité entre le fait et le but poursuivi par le législateur pour expliquer le choix retenu parmi plusieurs décisions possibles.

Il faut souligner qu'il existe une relation directe entre le type de compétence et la motivation. Plus la compétence est liée, moins exigeante est l'obligation d'exprimer les motifs de la décision. En cas de pouvoir discrétionnaire, dans la mesure où l'administration s'est vu attribuer un large pouvoir d'appréciation par le législateur dans la prise de décision (dans certains cas, elle peut prendre une décision parmi un certain nombre de solutions), elle sera tenue de donner plus d'explications pour rester dans la légalité propre à un État de droit, et dissiper toute ombre d'arbitraire. En conséquence, l'intensité de la motivation sera très forte dans une telle hypothèse.

Il convient également de souligner que la compétence n'est jamais totalement liée ni totalement discrétionnaire. Pour ce qui est de la compétence liée, même si le législateur a prévu qu'une fois certaines conditions vérifiées, l'administration devra agir d'une manière donnée, celle-ci aura une certaine marge de manœuvre, certes limitée, pour décider. Pour ce qui est du pouvoir discrétionnaire, même si le législateur n'a pas établi de limites quant au contenu de la décision, nous retrouvons des limites à d'autres égards. Ainsi en est-il de la compétence, en tant qu'autorisation légale d'agir conférée à l'administration. De même en est-il des barrières de protection érigées par les normes constitutionnelles et les règles de fond au soutien des droits des particuliers. Tout cela implique, dans le cadre d'un État de droit, la limitation du domaine de l'action de l'État à travers des garanties constitutionnelles reconnues aux citoyens.

Étant donné que la motivation constitue l'expression rationnelle d'un jugement, la pondération des faits et les antécédents sont les éléments qui pèsent le plus dans la décision car son traitement est nécessaire pour résoudre les questions proposées et l'administration ne doit pas être arbitraire.

La motivation se trouve en relation directe avec le droit à une procédure régulière. En effet, l'article ${ }^{\text {er }}$, paragraphe f, 3 de la LNPA garantit le droit du particulier d'obtenir une décision motivée et que «l'acte décisif considère expressément les principaux arguments et questions posées, eu égard à leur pertinence pour la résolution de l'affaire». Autrement dit, elle doit expliciter les raisons qui ont le plus pesé dans la décision.

L'intensité du contrôle juridictionnel du pouvoir discrétionnaire ayant évolué au fil des années, au nom de la légalité de l'action administrative, l'exigence de motivation s'est renforcée. En effet, traditionnellement, le contrôle du pouvoir discrétionnaire était limité. Cette limitation reflétait le caractère relatif des droits consacrés dans la Constitution nationale puisque leur exercice devait être «conforme aux normes qui réglementent [leur] exercice» (art. 14 de la Constitution nationale). En effet, la CSJN, dans l'arrêt de principe Cine Callao (1960) ${ }^{21}$, a reconnu à l'État le pouvoir d'intervenir pour la défense et la promotion des intérêts économiques de la société. Corrélativement, elle a interdit au pouvoir judiciaire de se prononcer sur l'efficacité et la convenance de la norme juridique, en restreignant le contrôle juridictionnel à la vérification de l'adaptation des 
moyens aux finalités c'est-à-dire au caractère raisonnable de la norme en ce qui concerne son application (mesures de police), d'après les idées d'Ernst Forsthoff sur l'acte administratif ${ }^{22}$.

Cette approche est en voie d'abandon. La loi et la jurisprudence tendent à faire de la motivation de l'acte un critère fondamental de sa légalité. Tel est le cas de la sanction de la loi 25.188 (1999) relative à l'éthique publique. En effet, l'article 2 précise que les sujets concernés par cette loi, parmi lesquels se trouve l'administration nationale, sont tenus de «e) Motiver leurs actes et de faire preuve de transparence dans les décisions prises, sauf exception tirée d'une norme ou de l'intérêt public».

Nous devons noter que, même si le contrôle juridictionnel est étendu, le juge ne pourra que prononcer l'illégalité de l'acte. Eu égard au principe de séparation des pouvoirs, celui-ci n'aura pas la possibilité de modifier la décision pour des raisons d'opportunité ou de convenance.

Compte tenu du système de contrôle juridictionnel des actes de l'administration en vigueur dans notre pays, nous pouvons indiquer que l'évolution de la jurisprudence ces dernières années tend à accorder une importance croissante à la motivation dans l'exercice d'un pouvoir discrétionnaire ${ }^{23}$.

Tout d'abord, dans l'arrêt Lema (2001), adopté à la majorité, la CSJN a jugé que l'exercice du pouvoir discrétionnaire ne dispensait pas d'

[...] observer l'élément essentiel [de la légalité de l'acte administratif] que constitue la motivation suffisante, étant donné que c'est justement dans ce domaine [du pouvoir discrétionnaire] de l'action administrative qu'elle se révèle le plus nécessaire encore ${ }^{24}$.

Plus tard, dans l'arrêt Schnaiderman (2008), la Cour a précisé, reprenant à son compte l'opinion de $\mathrm{M}^{\mathrm{me}}$ le procureur Laura Monti, que l'exercice du pouvoir discrétionnaire ne saurait être invoqué pour justifier l'action arbitraire d'un organisme administratif ni l'omission des exigences requises par la loi 19.549 pour la dictée de l'acte administratif, étant donné que la validité des actes des organes de l'État repose sur les principes de légalité et de l'action raisonnable et que ceux-ci permettent aux juges de vérifier l'existence desdites exigences, face aux questions présentées par l'intéressé.

Finalement, dans l'arrêt Silva Tamayo (2011), la CSJN a signalé que

[...] même s'il n'existe pas de formes rigides pour l'observation de l'exigence de la motivation explicite de l'acte administratif, la formulation de la motivation doit respecter le caractère particulier de chaque acte administratif; des formules sans contenu, des expressions très générales ou encore la simple énonciation des citations légales prévoyant un pouvoir générique non justifié dans des actes précis ne sont pas admissibles ${ }^{25}$.

Nous pouvons ainsi conclure que le contrôle juridictionnel de la motivation s'est accru, en passant de la non-intervention pour cause de pouvoir discrétionnaire à une intervention plus importante, en raison, précisément, de l'exercice d'un tel pouvoir ${ }^{26}$.

Cependant, il faut noter que la doctrine nationale a reconnu, tel qu'il a été signalé par Juan Carlos Cassagne, que certains actes exigent une motivation s'il s'agit de l'adoption de décisions visant des droits essentiels, tels que:

- des mesures d'exécution d'un jugement, comme dans le cas d'un redressement judiciaire;

- des décisions prises dans le cadre de l'exercice du pouvoir discrétionnaire;

- des actes abrogeant un autre acte pour lequel la motivation était exigée;

- des mesures limitant un droit subjectif, un droit collectif ou un intérêt diffus;

- des actes statuant sur un recours;

- des actes défavorables aux droits des particuliers ${ }^{27}$.

Dans tous ces cas, il s'agit d'actes qui ont été pris après l'accomplissement d'une procédure administrative formelle ayant généralement pour but l'octroi, la modification ou l'extinction d'un droit public subjectif.

\section{Conditions requises pour la motivation: le contenu de la motivation}

La LNPA précise les modalités requises pour la motivation des actes administratifs. D'après l'article 7, paragraphe e) de la LNPA, les éléments exigés prévus au paragraphe b) doivent y être présents, c'est-à-dire que l'acte administratif doit énoncer les faits et les précédents qui le justifient et les considérations de droit.

Justifier signifie donc donner les motifs pour ce qui a été décidé. C'est ainsi que, l'article 1, paragraphe f), alinéa 3 de la LNPA dispose que "l'acte de décision doit exprimer les principaux arguments et les questions proposées [par le requérant], en relation avec la résolution de l'affaire». Justifier ce qui a été décidé, sur la base des faits et des antécédents, signifie fournir des arguments sur les questions soulevées par l'édiction de l'acte.

Rafael Bielsa, dans son ouvrage de référence Derecho administrativo, signale qu'un acte est motivé en droit si

22. E. Forsthoff, Tratado de derecho administrativo, Madrid, Instituto de estudios políticos, 1958, p. 122

23. CNFed (Cámara Nacional de Apelaciones en lo Contencioso Administrativo Federal), chambre III, 8 mai 1984, Ubiría, Aníbal c. Estado nacional; chambre IV, 16 mars 1998, Negocios y Participaciones S.A. c. Dirección General Impositiva.

24. CSJN, 2001, Lema, décision 324:1860.

25. CSJN, 2001, Silva Tamayo, décision 334:1909.

26. P. Aberastury, La justicia administrativa, Buenos Aires, LexisNexis, 2006, p. 131 sq.

27. J. C. Cassagne, Derecho administrativo, t. II, p. 204 et doctrine citée. 
son dispositif est précédé de l'énonciation des raisons ou des fondements justifiant la décision par rapport à ses effets juridiques ${ }^{28}$.

Miguel S. Marienhoff a signalé que

[...] motif et motivation constituent des choses différentes; et il n'est pas possible d'assimiler la motivation au contenu, étant donné que la motivation n'est que l'expression des motifs (cause juridique) tandis que le contenu correspond à l'objet de l'acte. La motivation est un aspect ou un ingrédient qui relève de la forme de l'acte administratif. Elle ne constitue donc pas un élément autonome de cet acte. Elle traduit la juridicité de l'acte émis, en témoignant des considérations de fait et de droit qui justifient son émission. C'est ainsi qu'on s'éloigne de l'arbitraire: en somme, il s'agit d'une expression formelle en relation avec les circonstances de l'acte ${ }^{29}$.

La motivation peut être concise mais, pour être considérée valable, elle doit aborder les questions centrales sur lesquelles repose la décision. L'expression des motifs doit être effectuée d'une manière raisonnable et, d'après Marienhoff, se révéler suffisante, en raison d'une argumentation juridique cohérente.

Par conséquent, sur la base des principes de l'action raisonnable et de proportionnalité qui doivent être pris en compte dans le cadre de l'élaboration de l'acte, la doctrine nationale estime que la position de la Cour suprême relative aux jugements arbitraires peut être étendue aux actes administratifs. Ainsi, les principes régissant la prise de la décision judiciaire clôturant un procès sont applicables au moment du contrôle des actes administratifs.

Parmi les causes qui rendent un jugement arbitraire d'après la jurisprudence de la CSJN, Linares, en accord avec la majorité de la doctrine, a indiqué celles qui peuvent être appliquées à l'acte administratif. Il s'agit, à titre non exhaustif, du fait de:

a) ne pas statuer sur des questions substantielles soumises;

b) ne pas tenir compte de la norme légale et ne pas en faire état;

c) fonder l'acte sur des affirmations dogmatiques ou donner des arguments apparents. Nous devons inclure, dans ce cas, le détournement de pouvoir face à des buts non prévus par la loi $[\ldots]^{30}$.

La juridicité de l'acte n'est acquise que si le droit applicable énoncé dans la motivation constitue son fondement. Il existe donc une relation proportionnelle entre l'exercice du pouvoir discrétionnaire et l'exigence de la motivation. Plus l'exercice des facultés discrétionnaires est fort, plus l'argumentation doit être affinée, c'est-à-dire que la justification doit être plus approfondie; cet axiome est fondé sur la nécessité de respecter le principe de légalité et de passer avec succès le contrôle juridictionnel.

L’impartialité dans la prise de décision doit se révéler clairement dans l'expression de la motivation. En conséquence, il faut que les faits soient rationnels et adéquatement fondés et que l'évaluation du droit applicable soit accompagnée d'une argumentation juridique cohérente par rapport aux faits. Autrement, l'on tombe dans l'arbitraire.

La CSJN n'a pas appliqué la théorie du principe de proportionnalité du droit administratif allemand. Elle a retenu la notion de proportionnalité de la common law, selon laquelle le contrôle du caractère raisonnable est plus ou moins similaire au jugement d'adéquation (Geeignetheit). Néanmoins, la CSJN procède d'une façon plus abstraite qui n'exige pas la pondération des faits découlant de l'arrêt Wednesbury ${ }^{31}$.

La notion de proportionnalité élaborée par la CSJN établit que

[...] l'analyse de l'efficacité des moyens utilisés pour atteindre les fins visées par le législateur ne relève pas de la compétence de la Cour suprême, laquelle ne doit se prononcer que sur le caractère raisonnable des moyens choisis, c'est-à-dire décider s'ils sont proportionnels ou pas aux fins et, par conséquent, si la restriction aux droits individuels affectés est admissible ou pas ${ }^{32}$.

Cette opinion a été reprise dans l'arrêt Sindicato Argentino de Músicos, de 1960. Le tribunal y a remarqué qu'en matière de mesures de police, le caractère raisonnable doit être reconnu lorsque les mesures prises par l'autorité publique sont proportionnellement adéquates par rapport aux fins poursuivies par le législateur. Cette adéquation existe si l'acte étatique restreignant les libertés ne se révèle pas «évidemment et arbitrairement disproportionné ${ }^{33}$.

Nous pensons ainsi que le principe de l'action raisonnable comme fondement de la vérité ou de la justice ${ }^{34} \mathrm{com}$ porte aussi la proportionnalité entre les moyens employés et le but poursuivi; l'excès traduit le déraisonnable ${ }^{35}$. Par conséquent, la notion de proportionnalité utilisée par notre tribunal suprême n'appelle pas l'examen de la pertinence du critère adopté par le législateur dans l'exercice de ses compétences ${ }^{36}$. Elle implique l'examen des moyens choisis

28. R. Bielsa, Derecho administrativo, p. 33 .

29. M. S. Marienhoff, Tratado de derecho administrativo, t. II, p. 263

30. J. F. Linares, Fundamentos de derecho administrativo, Buenos Aires, Astrea, 1975, p. 322-323.

31. Associated Provincial Picture Houses Ltd v. Wednesbury Corporation [1947] EWCA Civ 1, [1948], 1 KB 223 (EWCA: England and Wales Court of Appeal; KB: King's Bench).

32. CSJN, $1^{\text {er }}$ septembre 1944, Pedro Inchauspe Hermanos, décision 199:483.

33. CSJN, 1960, Sindicato Argentino de Músicos, décision 248:80o.

34. J. F. Linares, Razonabilidad de las leyes, Buenos Aires, Astrea, 1970, p. 108.

35. M. S. Marienhoff, El exceso de punición como vicio del acto administrativo, Buenos Aires, La Ley, 1989-E, p. 969; M. S. Marienhoff, Tratado de derecho administrativo, t. II, p. 156.

36. D. J. Sesín, Administración pública. Actividad reglada, discrecional y técnica. Nuevos mecanismos de control judicial, $2^{\mathrm{e}}$ éd., Buenos Aires, LexisNexis, 2004, p. 349 
par rapport aux fins, afin de déterminer l'admissibilité de la restriction des droits individuels affectés ${ }^{37}$. Les arguments sont examinés de manière abstraite, sans faire référence aux faits pris en considération ${ }^{38}$. L'absence de pondération des faits a permis l'utilisation de la théorie du self-restraint ou d'auto-limitation judiciaire ${ }^{39}$.

L'application de la proportionnalité du droit allemand est bien différente, puisque celle-ci exige trois conditions: a) adéquation (Geeignetheit), b) exigence ou nécessité (Erforderlichkeit), et c) proportionnalité au sens strict; toutes les trois se traduisant par l'obligation de considérer des dérogations pour des situations exceptionnelles ${ }^{40}$.

Les actes qui accordent ou limitent les droits doivent aussi être motivés. C'est l'action de l'administration en tant qu'administratrice de l'intérêt public qui importe: elle doit agir en respectant cet intérêt, qu'il s'agisse d'un acte favorable ou défavorable aux intérêts des particuliers.

La motivation est donc contextuelle quand elle est exprimée dans l'acte administratif en cause. Mais la motivation peut également intervenir par renvoi à un autre texte, c'est-à-dire in aliunde; tel est le cas du renvoi à une opinion juridique donnée préalablement au cours de la procédure, ou encore par référence à une autre procédure, comme nous l'avons déjà signalé. Pour satisfaire à l'exigence de motivation, ce renvoi doit apparaître expressément dans l'acte.

\section{Les exceptions au principe de motivation des actes. Les actes automatisés}

La motivation est une règle générale, mais il faut aborder les exceptions à cette règle et vérifier si, à défaut de cette exigence, l'acte reste encore dans l'ordre juridique. Comme nous l'avons signalé, Marienhoff considérait que les ordres verbaux n'étaient pas visés par cette exigence. Cassagne évoque ainsi la situation d'une autorisation de passage donnée par un agent de police régulant la circulation au moyen de gestes: celle-ci est considérée comme un acte administratif parce qu'elle résout un problème et que sa violation entraîne une sanction administrative. C'est le cas aussi du changement de feu de circulation qui permet de continuer de rouler ou qui ordonne l'arrêt d'un véhicule, sans l'intervention humaine. Il en est de même de l'hypothèse, de plus en plus fréquente, où la décision résulte de l'application d'un algorithme.

L'article 7 de la LNPA prévoit la motivation en tant qu'exigence essentielle de l'acte administratif. Son absence entraîne la nullité absolue de l'acte par application de l'article 14, sans possibilité de régularisation ${ }^{41}$ (art. 14, 15 et 19 de la LNPA). L'acte sera légal seulement dans le cas où l'exception de non-motivation est posée par la norme.

Alors, nous devons analyser la portée de l'exception prévue à l'article 8 de la LNPA, qui est ainsi libellé:

L'acte administratif se manifestera expressément et par écrit, il fera mention du lieu et de la date de son édiction et il sera signé par l'autorité qui l'édicte; seulement par exception et si les circonstances le permettent, une forme différente peut être utilisée ${ }^{42}$.

Cette exception vient confirmer la règle générale et elle entraîne l'obligation de déterminer les circonstances dans lesquelles l'absence de motivation est possible, les cas où une forme différente peut être adoptée.

Il y a donc une quantité d'actes de la vie quotidienne de l'administration qui n'exigent pas l'expression des motifs ou qui sont automatisés. En matière éducative, un professeur peut noter un élève sans en indiquer les raisons, alors même qu'il émet un jugement de valeur dans l'exercice de son pouvoir discrétionnaire d'appréciation et que l'acte administratif que constitue l'attribution de la note remplit toutes les formalités requises par ailleurs. Cependant, il existe une obligation de communiquer, même verbalement, à l'élève les motifs de la notation, s'il en fait demande. Il s'agit d'une obligation éthico-morale. C'est le cas, par exemple, des règlements en vigueur à l'université de Buenos Aires, où cette formalité pour ce type d'actes n'est pas prévue. Malgré cela, en cas de demande formelle de l'élève, une procédure administrative est ouverte, et elle aboutira à l'émission d'un acte administratif motivé pour accepter ou refuser le désaccord. De même, le conducteur de l'automobile qui ne respecte pas le feu rouge commet une infraction. Les lois de la circulation routière admettent la constatation de ce type d'infraction, y compris par la voie électronique. Le constat est dressé par une procédure électronique sans l'intervention d'aucun fonctionnaire. Cependant, en cas de procédure administrative devant la justice administrative, cet acte administratif contesté doit respecter les exigences formelles et la décision finale doit être motivée.

Un autre aspect est à signaler. L'utilisation de l'administration électronique et des plateformes numériques permettent l'interaction avec l'administration, et elles offrent la possibilité de réaliser différentes démarches à travers les outils et les services disponibles en ligne, telles que la consultation des services, la prise d'un rendez-vous et l'accès à l'information. En somme, à partir de la mise en place des sites Internet uniques, la notion d'administration électronique entre en rapport avec celle d'intégration digitale des procédures internes et des services publics dans des écosystèmes digitaux ${ }^{43}$.

37. CSJN, 1984, Bignone, décision 306:655; CSJN, 1978, Timerman, décision 300:816.

38. P. Aberastury, La justicia administrativa, p. 93.

39. G. Carrió, «Nota sobre el caso de los números vivos », Revista del Colegio de Abogados de La Plata, année 3, n 6, 1961, p. 49-76.

40. E. Schmidt-Assmann, La teoría general del derecho administrativo como sistema, Madrid, Marcial Pons, 2003, p. 349-350.

41. Le retrait d'un acte irrégulier créateur de droits ne peut intervenir que par voie juridictionnelle.

42. Nous soulignons.

43. Voir «Carta iberoamericana de gobierno electrónico», Centro Latinoamericano de Administración para el Desarrollo, Pucón (Chili), 31 mai et $1^{\text {er }}$ juin 2007, p. 24, cons. 30, en ligne: http://old.clad.org/documentos/declaraciones/cartagobelec.pdf/view; J. G. Corvalán, «Gobierno y 
En outre, l'utilisation des systèmes d'intelligence artificielle au service de l'administration publique entraîne l'apparition d'un nouveau défi, tel que signalé par $\mathrm{M}^{\mathrm{me}}$ la professeur Diana Urania Galetta, et illustré par le traducteur de Google, le système informatique d'intelligence artificielle Watson développé par la société américaine IBM qui est en mesure de répondre à des questions posées dans un langage naturel ou même le système d'intelligence artificielle de prédiction au service de la justice, Prometea, mis en marche par le Ministère public de la ville de Buenos Aires en 2017, qui permet l'élaboration des avis juridiques de manière intégrale ${ }^{44}$.

Sera-t-il possible d'appliquer les caractéristiques déjà mentionnées de la motivation à une décision prise digitalement et sans intervention d'une personne humaine? Où est la limite? Il s'agit d'un défi pour les générations à venir.

Nous sommes en mesure de signaler donc que l'absence de motivation au moment de l'émission de l'acte constitue une circonstance exceptionnelle qui n'admet pas seulement la révision de l'acte mais aussi sa contestation en raison de l'absence de motivation requise, notamment en cas d'ouverture d'une procédure formelle par le particulier ${ }^{45}$. L'exception peut être prévue par le législateur ou intervenir en cas de décision prise dans le cadre d'une procédure digitale. Tel est le cas des acquisitions électroniques qui automatisent la décision en fonction des données fournies au système électronique.

En droit argentin, la doctrine et la jurisprudence ont imposé le devoir de l'administration de motiver les actes administratifs. Elles se sont également prononcées sur le type d'acte soumis à motivation ainsi que sur les modalités de la motivation.

L'adoption du principe général de la motivation de tous les actes administratifs définitifs est un principe sain qui permet un plus grand contrôle, soit administratif soit juridictionnel. Néanmoins, il existe des actes administratifs qui n'exigent pas la motivation au moment de leur émission. Par conséquent, il faut faire une distinction entre la plupart des actes et ceux qui, par exception, peuvent se manifester sous une forme différente, ou les actes pour lesquels la norme prévoit expressément que la motivation n'est pas nécessaire, ou encore les actes provenant de l'application d'algorithmes sans qu'aucun fonctionnaire n'ait à intervenir pour leur émission.

Comme nous l'avons signalé, la décision de l'administration peut être soumise au contrôle au titre de la motivation, et ce contrôle couvre tout type d'acte. Tel est le cas des actes émis à la suite d'une procédure à issue favorable ou défavorable pour le particulier et, notamment, afin d'éviter l'arbitraire, les actes édictés dans le cadre du pouvoir discrétionnaire.

Les classifications fondées sur le contenu de ce qui relève de la décision ne seront pas utiles étant donné qu'elles ne comprennent pas tous les cas et que le législateur ne peut pas prévoir toutes les possibilités. Par conséquent, le droit administratif argentin propose la motivation comme une question formelle. La détermination des circonstances permettant l'adoption d'un acte administratif sans aucune motivation est bien difficile.

Les exemples proposés relatifs au feu rouge ou à l'appréciation du travail de l'élève ont en commun l'inexistence ou l'absence de nécessité de remplir une procédure formelle avant l'émission de l'acte. Nous considérons alors que si l'émission de l'acte n'est pas soumise à une procédure formelle, celui-ci peut être édicté sans motivation.

Néanmoins, dans le cas exceptionnel d'absence de motivation, si l'intéressé exprime son désaccord, l'administration devra lui communiquer, de manière suffisante et même ex post facto, les raisons de sa décision. Par contre, l'acte administratif pris dans le cadre d'une procédure formelle en l'absence de motivation sera vicié et donc illégal, sans possibilité de reformulation postérieure. Corrélativement dans ce dernier cadre, si la procédure ne prévoit pas formellement la possibilité de procéder sans motivation, au vu des circonstances, l'acte sera en principe légal, mais, en cas de désaccord, l'administration devra motiver.

Au cas où la norme ferait uniquement mention des situations où la motivation est exigée pour la validité de l'acte (tel est le cas du système français), il faudra analyser s'il s'agit d'une énumération exhaustive ou pas.

Pour conclure, si la norme prévoit l'obligation de la motivation de l'acte, l'exception sera admise seulement dans certaines circonstances dûment prévues par les textes ou si la nature de l'acte le permet; cependant, même si la norme admet la non-motivation de l'acte, l'administration reste obligée de justifier a posteriori son action à travers une décision motivée.

Le principe de légalité régissant toute procédure administrative impose à l'administration de donner les explications et les justifications nécessaires et suffisantes pour assurer la transparence de son action.

administración pública digital. Enfoque internacional (ONU, OEA y CLAD) y su vinculación con la República Argentina», Revista jurídica de Buenos Aires, année 43, nº 96, 2018, p. 17-50.

44. D. U. Galetta, «Digitalización y transparencia : ¿ un "responsable de la transparencia” y su "asistente digital” como herramientas del buen gobierno del futuro?», Revista jurídica de Buenos Aires, année 43, n 96, 2018, p. 159-180.

45. La Procuración del Tesoro de la Nación (PTN), organisme de consultation juridique du pouvoir exécutif national et dont la doctrine doit être suivie par les avocats qui plaident au nom de l'administration nationale, a exposé des principes similaires à ceux que nous présentons dans cette étude, à savoir : «Un principe directeur en matière de procédure administrative impose à l'administration le devoir de résoudre les réclamations et les questions posées par les particuliers à travers des décisions motivées, conformément au droit à une procédure régulière, prévu dans l'article $1^{\circ}$, paragraphe f), de la LNPA» (opinion PTN 249:505, 3 juin 2004). 